\title{
"De la interoperabilidad de la bolsita a la Cobertura Universal de Salud". Un análisis sobre la agenda política y los sentidos de los sistemas de información
}

\author{
"From the interoperability bag to Health Universal Coverage". An \\ analysis of governmental agenda and meanings of informational
} systems

Daría Belén Lopez Castro

lopezcastromb@gmail.com

Facultad de Filosofía y Letras, Universidad de Buenos Aires, Argentina

Recepción: 01 Octubre 2020

Aprobación: 30 Septiembre 2021

Publicación: 01 Diciembre 2021

Cita sugerida: Lopez Castro, M. B. (2021). "De la interoperabilidad de la bolsita a la Cobertura Universal de Salud”. Un análisis sobre la agenda política y los sentidos de los sistemas de información. Cuestiones de Sociología, 25, e127. https://doi.org/10.24215/23468904e127
Resumen: Una integración artesanal de las trayectorias de salud personales suele ser hecha por los pacientes, generalmente reuniendo sus estudios clínicos en las típicas bolsas de análisis. Los recursos informáticos del presente permiten que lo que con humor los médicos llaman la "interoperabilidad de la bolsita" se resuelva a partir de recursos digitales compartidos en el territorio nacional. El objetivo del presente trabajo es identificar y analizar el tratamiento de la interoperabilidad de los sistemas de salud en la agenda política, así como sus múltiples sentidos. Se recurrirá al enfoque del ciclo de las políticas públicas para estudiar a los actores y los procesos involucrados. Se argumentará que la comunidad de especialistas en informática en salud, junto a la propuesta de organismos internacionales sobre la Cobertura Universal fueron fuerzas que traccionaron el tratamiento ofreciendo una solución tecnológica que resuelve favorablemente los intereses de los actores involucrados.

Palabras clave: Políticas Públicas, Informática en Salud, Cobertura Universal de Salud, Teoría Crítica de la Tecnología.

Abstract: A craft integration of personal health records is usually done by patients gathering their clinical studies in the typical results bags. Computational resources of the present allow what doctors humoristically call the "bag interoperability" be solved by digital resources shared in the national territory. The objective of this paper is to identify and analyze the treatment of information health systems' interoperability as an object of governmental agenda, as well as its multiple meanings. The public policy cycle approach will be used to study the actors and processes involved. It will be argued that the community of specialists in health informatics, together with the proposal of international organizations on Universal Health Coverage were forces that brought the issue to the agenda, offering a technological solution that favorably resolves the interests of the actors involved. Key words: Public Policies- Health informatics- Universal Health Coverage- Critical Theory of Technology Introducción

Keywords: Public Policies, Health informatics, Universal Health Coverage, Critical Theory of Technology. 


\section{Introducción}

Una integración artesanal de las trayectorias de salud personales suele ser hecha por los pacientes, generalmente reuniendo sus estudios clínicos en las típicas bolsas de análisis. Esta integración depende de la organización y la voluntad individual de los ciudadanos e incluye solo los registros clínicos a los que tienen acceso. Esta situación que los especialistas llaman con humor la "interoperabilidad de la bolsita" puede remplazarse mediante la digitalización de los registros y con la implementación de Sistemas de Información en Salud (SIS) que permitan integrar los datos de salud.

Mientras que algunos ciudadanos tienen una bolsa donde guardan y trasladan sus estudios de salud complementarios, otros hacen uso de los dispositivos digitales para obtener turnos y comunicarse con los equipos de profesionales lo que genera diferencias en las formas de atención entre unos y otros. Una red nacional de información en salud permitiría no depender de la capacidad de almacenamiento de los pacientes y mejorar la respuesta de urgencias. El sistema de salud nacional no se encuentra integrado ni totalmente digitalizado, a pesar de los esfuerzos de los prestadores de salud. La disponibilidad de información mejora la atención de la salud (Friedman, 2009), sin embargo, unos años atrás acceder a la información sobre la trayectoria de salud de un paciente atravesando los diferentes subsistemas (público, privado o de la seguridad social) y las jurisdicciones no era siquiera un proyecto. De un tiempo a esta parte, la incapacidad para disponer de datos en tiempo real sobre los pacientes se construyó como un problema de salud y la falta de políticas públicas que impulsen estos proyectos como un problema social por parte de un grupo de profesionales.

Las tecnologías que permiten acceder a los registros de salud en tiempo real sin depender de la voluntad y capacidad de almacenamiento de los pacientes existen desde hace tiempo. Los desarrollos de registros electrónicos en el ámbito de la salud se remontan a los años 60 (Lindberg, 1965). La misma antigüedad tiene la interoperabilidad, es decir, la capacidad de comunicación entre distintos SIS. Los primeros estándares para mensajería se remontan a los años 60, y en el año 1987 se conformó Health Level Seven (HL7), una de las sociedades con mayor influencia (Simborg, 1988). A pesar de la disponibilidad técnica, en Argentina, la comunicación entre SIS más frecuente es aquella que llaman "interoperabilidad de la bolsita” (Rizzato Lede, 2018). A lo largo de los años se han llevado adelante diversas iniciativas para digitalizar e integrar los registros de salud desde diferentes prestadoras y sociedades de profesionales. Sin embargo, fue recién en 2016 que el tema logró instalarse en la agenda política. En diciembre de 2018 se publicaron en el Boletín Oficial los estándares para la interoperabilidad y se delegaron a la Dirección Nacional de Sistemas de Información de Salud (DNSIS) las facultades para realizar las actualizaciones necesarias.

Si la disponibilidad de información sobre la trayectoria de salud mejora la atención ¿por qué no se desarrolló un plan nacional de acceso en tiempo real a los datos de salud con anterioridad?, ¿por qué el desarrollo de una estrategia 
nacional de salud digital no logró la demanda amplia y movilización de la sociedad civil? Ambas preguntas fueron disparadoras en el planteo del presente trabajo, aunque exceden los objetivos de este artículo. Aquí nos proponemos analizar el ascenso y tratamiento de la salud digital en la agenda de las políticas públicas. Para ello tomamos como hito la publicación de los estándares de interoperabilidad en el Boletín Oficial, considerando que se trata de la primera medida de una estrategia para el despliegue de una serie de políticas articuladas sobre SIS. Así, identificaremos los actores y procesos que habilitaron el tratamiento de la interoperabilidad en la agenda desde el enfoque del ciclo de las políticas públicas.

En primer lugar, dedicaremos dos apartados a explicitar las formas en que construimos el problema de investigación. En "El ascenso de una tecnología como tema de la agenda política" señalaremos el enfoque teórico metodológico elegido para el presente trabajo introduciendo la propuesta del modelo de John Kingdon y la noción de código técnico de Andrew Feenberg. Seguidamente, en "Notas sociotécnicas sobre la interoperabilidad de los SIS", definiremos la interoperabilidad como tecnología y recuperemos el trabajo de Paul Edwards para señalar sus implicaciones sobre las formas de organización de la medicina. En segundo lugar, desplegaremos el análisis del ascenso de la interoperabilidad en la agenda política siguiendo a Kingdon en tres apartados. Iniciaremos por identificar el lugar de los informáticos en salud como grupo de interés y su logro en la creación de una Dirección Nacional de Sistemas de Información en Salud como un agente gubernamental capaz de movilizar su agenda. Luego, describiremos los flujos de procesos involucrados señalando el rol de la Cobertura Universal de Salud propuesta por la Organización Mundial de la Salud y el cambio de gobierno. Por último, volveremos a articular toda la descripción y análisis realizado para señalar el tipo de ventana de oportunidad que permitió finalmente el tratamiento de la interoperabilidad como una política pública.

\section{El ascenso de una tecnología como tema de la agenda política}

Para varios científicos sociales las políticas públicas se presentan como una vía de acceso al estudio del Estado. Para nosotros, el análisis de la agenda política resultó ser una estrategia para analizar las relaciones entre una serie de actores a los que seguimos (Latour, 2008) en el marco de un proyecto de investigación desde el enfoque de la antropología de la ciencia y tecnología. El objetivo es dar cuenta de la emergencia de un nuevo campo disciplinar: la informática en salud. De este modo, el análisis de las políticas públicas en salud digital permitió resignificar las relaciones y las alianzas entre diversas instituciones y el Estado.

Para este artículo, se revisitó el corpus generado durante el trabajo de campo etnográfico que contempla la reconstrucción de las trayectorias sociotécnicas de una serie de SIS y las trayectorias de profesionales del campo. El material empírico utilizado para este artículo es el producto de entrevistas a informáticos en salud y de observación participante realizada en eventos de la comunidad de profesionales (como espacios de formación, congresos y comunicación de medidas estatales) entre julio de 2016 y enero de 2020. También se ha analizado la producción científica de los profesionales entrevistados, los informes de organismos públicos e internacionales, las resoluciones ejecutivas publicadas en el Boletín Oficial y artículos periodísticos. El material fue compilado y analizado 
desde un enfoque cualitativo. La codificación del material respondió a las categorías de análisis del proyecto principal relacionadas con la construcción de la informática en salud como disciplina, del perfil profesional de los informáticos en salud y los tipos de relación con diversas instituciones. El modelo del ciclo de las políticas públicas permitió resignificar el análisis.

Partir desde las políticas públicas para analizar las alianzas que establecen quienes participan en la construcción de una nueva disciplina es posible a partir de las definiciones relacionales del Estado, y también a las posiciones que sostienen que las políticas públicas implican una relación causal donde múltiples actores se movilizan, deciden y ejecutan soluciones frente a una cuestión social problematizada (Oszlak \& O’Donnell, 1995). Según Oszlak y Gantman (2007) el Estado existe mientras tenga cuestiones por resolver, de modo que la definición de los problemas y sobre quiénes participan como expertos capaces de proponer soluciones al Estado responde al resultado de diversos agentes con intereses movilizados (Epstein, 1996; Jasanoff \& Simmet, 2017).

En este artículo tomamos el modelo elaborado por John Kingdon (2014) para analizar no solo el rol de los "actores" sino también el "flujo de procesos" que constituyen el contexto de acción. La propuesta de este autor combina elementos de las teorías del "ciclo de las políticas públicas" y el modelo de "lata de basura". Esto permite hacer foco en el proceso de fijación de los temas en la agenda, es decir, en el momento previo a la implementación de los programas de gobierno. Este autor propone realizar dos operaciones: identificar a los actores involucrados y distinguir su filiación, gubernamental o extra-gubernamental, y reparar en los "flujos de procesos" que impulsan este tipo de cuestiones, (1) la construcción de problemas o crisis, (2) la presentación de alternativas por parte de comunidades de especialistas y (3) el humor político del momento.

Un problema socialmente relevante para la sociedad civil puede no ser objeto de atención del Estado (Oszlak, 2006), así como un objeto de atención política puede no ser de interés para la sociedad civil. Así, reparar en la apertura de una "ventana de oportunidad" para el tratamiento y legislación sobre un tema permite reorganizar la mirada sobre la construcción de las agendas y analizar el peso relativo de los actores en medio de procesos sociales más amplios.

Junto al modelo de Kingdon, recuperamos la noción de código técnico de la teoría crítica de la tecnología propuesta por Andrew Feenberg. Desde esta categoría, la interoperabilidad de los SIS como solución técnica al problema de la disponibilidad de información en salud es la realización de los intereses de diversos actores (Estado, informáticos en salud, financiadores y prestadores de salud, sanitaristas, organizaciones ciudadanas). Así, se configura como una opción, entre otras alternativas, al dar respuesta a diversas necesidades. A la vez, la interoperabilidad condensa diversos sentidos para cada uno de los involucrados, ya que las tecnologías son inherentemente políticas (Winner, 1980) y su elección en el marco de proyectos estatales evidencian aún más las formas de poder y de organización social que coproducen (Jasanoff, 2004).

\section{Notas sociotécnicas sobre la interoperabilidad de los SIS}

Nuestro interés radica en las políticas públicas de lo que se llama salud digital o cibersalud. Para este trabajo nos centramos en el asenso y tratamiento de 
la interoperabilidad de los SIS porque fue presentada como el eje central que organiza la forma en que el Estado argentino desplegará los desarrollos informáticos. Partimos de la premisa de que la interoperabilidad es una tecnología, y como tal el resultado tanto de procesos sociotécnicos. Al ser el objeto central de una política pública, podemos dar cuenta de cuál es la visión que el Estado le otorga, y cómo los expertos la utilizan para imaginar un futuro (Jasanoff $\&$ Kim, 2013) con una infraestructura nacional de información en salud. A continuación, describiremos algunas cuestiones que nos parecen importantes para comprender por qué logra protagonismo en la agenda política sobre SIS.

La interoperabilidad puede ser definida como "la capacidad de dos o más componentes de software para cooperar a pesar de las diferencias en el lenguaje, la interfaz o la plataforma de ejecución" (Wegner, 1996). De modo más llano, podemos decir que es la capacidad de dos sistemas de intercambiar información. Una alternativa técnica para la construcción de una red integrada es el diseño y desarrollo de un SIS monolítico, es decir, de un único sistema. Este tipo de SIS donde el lenguaje de programación y la plataforma de ejecución serían únicos harían irrelevantes discutir estándares de interoperabilidad. Sin embargo, esta solución suele ser descartada debido al alto nivel de inversión que requiere y la posibilidad de quedar obsoleto por la actualización de la tecnología en el corto plazo. Lo más frecuente es el desarrollo de sistemas modulares y que estos se comuniquen gracias al uso de estándares.

En todos los ámbitos (aviación, finanzas, ciencias de la atmósfera, etc.) los debates respecto a la interoperabilidad suelen darse puertas adentro en comunidades de prácticas reducidas. Dado el alto contenido técnico de estas discusiones, quedan relegadas a un pequeño grupo de expertos. En el campo de la salud, las discusiones sobre la interoperabilidad también implican revisitar las cuestiones sobre las políticas de seguridad, privacidad, propiedad y custodia de la información clínica (Herrero y Guerra, 2017), así como el tipo de registro y de visualización de los datos que tendrán los profesionales de la salud, el personal administrativo y/o los pacientes (Gunter \& Terry, 2005). De este modo, las decisiones y la definición de estándares suponen más que una discusión sobre la disponibilidad de tecnología.

Paul Edwards (2010) analizó la determinación de estándares en las ciencias de la atmósfera. Argumentó que la creación de un sistema de información planetario implicó dos procesos: por un lado "hacer los datos globales" (making global data) y por el otro "hacer globales los datos" (making data global). El resultado del despliegue de instrumentos para la medición de valores y la estandarización de los datos permitió el desarrollo de modelos predictivos sobre la atmósfera y el clima. También, implicó el surgimiento de una nueva forma de pensamiento, una "conciencia planetaria" donde la atmósfera es un sistema interdependiente.

Recuperamos a este autor ya que identificamos que ambos procesos tienen similitudes con la infraestructura que propone la informática en salud. En un primer momento, "hacer los datos globales" implicaría la implementación de sistemas digitales que habiliten el ingreso estructurado del registro médico. Este proceso implica varias dificultades porque supone la estandarización de prácticas médicas, o cuando menos de los términos utilizados. La variabilidad de prácticas médicas y de las lógicas de racionamiento han mostrado ser un obstáculo en la implementación de SIS (Berg, 1997) junto con la ambigüedad del vocabulario. 
El segundo proceso, "hacer globales los datos", permite que el ingreso estructurado de datos logrado en la etapa anterior alimente sistemas de soporte a la toma de decisiones. Estos SIS son la mayor de las promesas de los registros electrónicos. Los sistemas de soporte no solo colaboran en la vigilancia epidemiológica y la gestión de los centros, sino que pueden asistir en la reducción del error médico en el punto y momento de atención (Luna et al., 2015). Combinados con avances de la genética se habilita el despliegue de la medicina personalizada, de precisión (Castaneda et al., 2015) y predictiva. Así, las redes integradas de información en salud tienen el potencial de modificar el actual régimen médico (Clarke, Shim, Mamo, Fosket \& Fishman., 2003).

$\mathrm{Al}$ analizar estos procesos de captura, transmisión, copia, almacenamiento y recuperación de los datos, Edwards empleó la metáfora de la "fricción" para describir la resistencia que se produce a lo largo de las distintas superficies por las que transita el dato. La automatización busca reducir la fricción, pero implica un gran consumo de energía al requerir de la creación de estándares a través del consenso. Ahora bien, los estándares de interoperabilidad en salud no se encuentran totalmente estabilizados. Si bien hay un consenso generalizado para el desarrollo de sistemas modulares, la construcción de consenso sobre la interoperabilidad continúa consumiendo energía. La sociedad cuyos estándares tienen mayor influencia actualmente en el ámbito de la salud es Health Level Seven (HL7), pero también tienen presencia organizaciones como International Standards Organisation (ISO), Comité Européen de Normalisation (CEN) y OpenEHR.

Revisar la definición de interoperabilidad junto al trabajo de Edwards, permite reconocer que la elección de estándares tiene implicancias que van más allá de las fronteras de un país conformando infraestructuras que organizan nuevos regímenes de salud. A otro nivel, también nos advierte que los Estados deben elegir estándares de interoperabilidad y de terminología para desplegar redes integradas.

El Estado Argentino había avanzado en los procesos para "hacer los datos globales" y "hacer globales los datos" a partir de los esfuerzos de diferentes dependencias dentro de los organismos gubernamentales. En 2007, se dio inició al proyecto hoy llamado "Sistema Integrado de Información Sanitaria Argentino" (SISA) con el propósito de crear un repositorio a partir de la digitalización de las "transacciones realizadas por los usuarios/ciudadanos en el sistema de salud, sea a partir de los actuales mecanismos de registro y comunicación de datos, como a partir de futuros desarrollos tales como la 'Ficha Única del Ciudadano' o la 'tarjeta sanitaria'”(Ministerio de Salud, 2007). Inicialmente este proyecto concentró la información reportada por los centros de salud a otros programas como el Sistema de Vigilancia Epidemiológica sobre SIDA o el Sistema Nacional de Información Perinatológica. Junto a SISA, se destaca la Red Federal de Telesalud creada en 2014. El despliegue de la infraestructura para su desarrollo fue posible gracias a la empresa de telecomunicaciones del Estado Arsat.

Dentro del ámbito del Ministerio de Salud se había conformado una unidad de interoperabilidad que consistía en reuniones periódicas con representantes de los diversos organismos y proyectos. El objetivo de estas reuniones transversales al organigrama era mejorar la comunicación entre los programas e intentar articular 
los distintos desarrollos informáticos. La función de este espacio pasó a ser de la Dirección Nacional de Sistemas de Información en Salud creada en 2018, uno de los actores centrales en la definición de la agenda que describiremos a continuación.

\section{Los actores relevantes. El especialista para la interoperabilidad en salud}

El primer paso que propone el modelo de John Kindon para analizar las políticas públicas es identificar los actores, dentro y fuera del gobierno, involucrados en la movilización del tema en la agenda política (2014, pp. 21-70). Esta primera etapa invita a identificar el rol de: (1) presidentes y sus equipos, (2) los parlamentos y de los equipos de los legisladores, (3) los empleados estatales, (4) los medios de comunicación, (5) de la opinión pública, (6) los grupos de interés y (7) la academia. El trabajo por posicionar a la interoperabilidad en la agenda no fue iniciado por un grupo de interés. Los informáticos en salud comenzaron a movilizar su agenda de investigación y desarrollo como parte de la institucionalización de la disciplina.

Los profesionales del emergente campo de la informática en salud han sido quienes construyeron como problema la falta de disponibilidad de datos de salud en tiempo real. Entendemos que esta capacidad para "enmarcar" (to frame) la realidad y señalar sus problemas son modos de intervención política. Steven Epstein (1996) argumentó que los marcos interpretativos no solo permiten delimitar aquello que merece ser atendido y destacado de la realidad, sino que habilitan la construcción de alianzas, obtener adherentes y desmovilizar a los antagonistas.

A mediados de los años 70 surge la informática en salud como una diciplina cuyo objetivo era reparar en el registro, almacenamiento, recuperación y uso óptimo de los datos para la resolución de problemas y la toma de decisiones. Los primeros desarrollos informáticos aplicados al ámbito asistencial tuvieron lugar en contextos de experimentación universitaria, gracias a su diseminación en los últimos años, se hace difícil no encontrar un área de desarrollo propio o de asesoramiento para la compra de software en los centros asistenciales. En el ámbito nacional, la comunidad de especialistas está mayormente conformada por profesionales que han aprendido su quehacer en la práctica cotidiana, ya que recién en los últimos años se acreditaron ofertas de educación relacionados a este campo de conocimiento. La ampliación de la demanda laboral junto a las nuevas posibilidades de formación habilitó el crecimiento exponencial de la comunidad que se había mantenido reducida por dos décadas.

La construcción de las fronteras de la informática en salud implicó señalar las particularidades del campo de la salud frente a otros espacios de implementación de sistemas de información. Paradójicamente, desde el discurso la forma en que se construyen como expertos en SIS no es haciendo foco en la dimensión tecnológica, sino que han corrido el eje de los debates hacia el acto médico. Así, buscan que la cuestión tecnológica sea el medio y no el fin de la planificación y desarrollo (Gardner et al., 2009; Mantas et al., 2010).

Es importante recordar que el proyecto de salud digital es un proyecto de salud, es un plan pensado desde la salud pública y no desde lo frío de los sistemas. (Notas de 
campo, 2019. Funcionario presentando el plan nacional de salud digital en un evento organizada por FMED, UBA).

La solución a todo no es la tecnología, pero la gestión tecnológica de la información puede cambiar mucho en el día a día de la salud pública. La limitante es la situación económica y que no haya voluntad política para que las instituciones funcionen en red. (Notas de campo, 2017. Entrevista a médica informática en formación).

(E)l que no entiende que la informática en salud es la nueva forma de hacer salud pública no entendió nada. (Notas de campo, 2017. Entrevista a Médica informática en formación).

La informática en salud dialoga constantemente con otras disciplinas (Lopez Castro, Smulski \& Hidalgo, 2019), lo que les brinda herramientas a los informáticos para señalar a la dimensión social y política de la tecnología.

A nivel internacional se reconoce la experticia de los informáticos en salud sobre los SIS. Organismos internacionales, como la OPS/OMS, han informado sus decisiones en recomendaciones de los nuevos especialistas. De hecho, los referentes nacionales del campo participan de modo activo en el diseño de investigaciones y programas de la OPS/OMS que luego informan las políticas públicas de los Estados de la región.

A lo largo de los años, miembros del campo de la informática en salud han acercado propuestas para el desarrollo de una red integrada de información al poder ejecutivo y a los equipos de los legisladores sin lograr que este proyecto se concretara.

Ya en los '90 le habíamos acercado proyectos a diputados (...). Lo mismo hicimos más adelante, por eso, nos llamaron para armar el presupuesto para una red nacional (...) en ese momento no era nada en dinero, se podría haber hecho. ¿Te imaginás lo que hubiera significado? ¿los problemas que nos hubiéramos ahorrado? (Notas de campo, 2016. Entrevista a Informático en Salud).

Los proyectos acercados a los políticos han sido variados, presentado diversas alternativas; sea porque se fueron actualizando en función de las tecnologías disponibles o porque dentro de la comunidad existen diferentes posiciones respecto a cómo debe llevarse adelante el desarrollo de un SIS y cuál es el rol del informático en salud en las instituciones. En 2016, ante la apertura de una ventana de posibilidad para el avance de las políticas de salud digital, al igual que otras comunidades, moderaron sus posiciones para que la iniciativa tenga oportunidades de implementación (Kingdon, 2014, pp. 172-179). Así, la reducción de la fragmentación interna resultó estratégica.

La ventana de posibilidad permitió la creación de la Dirección Nacional de Sistemas de Información en Salud (DNSIS) dentro del ámbito de la Subsecretaría de Coberturas Públicas Sanitarias del Ministerio de Salud Nacional (Ministerio de Salud, 2018). La creación de la Dirección y la elección de un médico informático para su conducción evidencian un reconocimiento del Estado a la disciplina y la experticia de la comunidad local. A la vez, permite la consolidación y creación de nuevos espacios de trabajo. Mientras los prestadores de salud deberán consultar a los especialistas para adecuarse al marco de las nuevas disposiciones, también los informáticos en salud podrán acercar a la DNSIS los intereses y necesidades de las instituciones que los empleen.

La DNSIS ha sido el organismo gubernamental creado para llevar adelante el desarrollo de un plan nacional de salud digital y, por lo tanto, actor principal en 
el posicionamiento político de la interoperabilidad. Los proyectos relacionados a telesalud y SISA quedaron dentro de la órbita de la flamante dirección cuyo objetivo es orientar el desarrollo y expansión de los sistemas de información como una estrategia sanitaria. Conformada por coordinadores técnico-políticos y un equipo de empleados del ministerio reasignados a la nueva dirección, se abocó a analizar las alternativas posibles en términos de desarrollo tecnológico y viabilidad que prestadores y financiadores de salud nacionales acompañen las medidas.

Entre las primeras medidas, en enero de 2018 la DNSIS optó por asociarse a SNOMED, un consorcio internacional que elabora y publica estándares de terminología clínica (Ministerio de Salud y Acción Social, 2018). A partir de esta medida, el Ministerio recomendó incluir el estándar en las aplicaciones que se desarrollaran y comenzó a brindar capacitación y soporte a los prestadores y a las jurisdicciones que adhieran al plan nacional. El uso de SNOMED garantiza que los datos se ingresen estructurados, reduciendo la ambigüedad y facilitando su disponibilidad. Además del estándar terminológico, la DNSIS se encaminó a definir un estándar de comunicación.

Para elegir estándares de interoperabilidad la DNSIS tomó un modelo de trabajo frecuente en el diseño de software. Se generaron reuniones de consenso con representantes de las jurisdicciones provinciales, de las farmacéuticas, prestadores y financiadoras de salud para realizar pruebas de concepto y colaborativamente buscar soluciones al desarrollo. Así, la decisión de la DNSIS fue conocer cómo trabaja cada jurisdicción e instituciones y buscar alinear los esfuerzos preexistentes en lugar de definir un modelo único y centralizado. Se mantuvieron las formas de producción y almacenamiento de los datos de cada prestador y se optó por la creación de un "Bus de Interoperabilidad" que otorgaría "la posibilidad de dar funciones para la indexación y localización de documentos clínicos en una arquitectura nacional de repositorios clínicos distribuidos, a cargo de cada una de las instituciones que genera el dato" (Resolución N. ${ }^{\circ} 115$, 2019). Esto implica que la DNSIS es responsable de desarrollar y mantener la arquitectura de una plataforma con bases de datos y servidores que se adaptan a la organización del sistema de salud nacional. El Bus permite acceder a datos y sistemas prexistentes y exige el uso del estándar HL7 FHIR.

La interoperabilidad como código técnico habilita señalar los intereses y sentidos que condensa para cada actor involucrado. Para la comunidad de informáticos, la interoperabilidad es una alternativa de desarrollo. Esta opción tecnológica como política estatal reconoce los trabajos realizados con anterioridad al elegir un estándar que venía utilizando la comunidad. Para el Estado, la interoperabilidad supone una baja inversión frente a otras alternativas de desarrollo de una red. La creación y mantenimiento de un Bus de Interoperabilidad le permite articular los esfuerzos y desarrollos previos de aplicaciones e infraestructuras como SISA y la red de telesalud. A la vez, reduce las fricciones y el desgaste que supondría imponer un modelo único a las jurisdicciones, prestadores, financiadores y farmacéuticas del sistema. Presentada como una opción surgida de espacios de trabajo en consenso con estos actores, la interoperabilidad se ajusta a las prácticas actuales. 


\section{Los flujos de procesos. Los SIS como puente para el acceso a la salud}

Además de identificar a los actores civiles y gubernamentales involucrados en el tratamiento de un tema, Kingdon propone identificar los "flujos de procesos" que facilitan u obstruyen su tratamiento político. La identificación y análisis de los procesos relevantes para una política pública presenta desafíos metodológicos. $\mathrm{Al}$ identificar que la construcción de la agenda es el resultado de múltiples factores y del accionar de grupos con diferencias internas cualquier descripción puede parecer reduccionista. De igual modo, la reconstrucción de procesos para su análisis podría presentar al Estado y sus políticas como resultado de procesos incrementales y racionales, cuando se trata de procesos desordenados. Siguiendo el modelo de la lata de basura, Kingdon (2014, pp. 71-89) reconoce que las políticas públicas son producto de la mezcla de problemas, soluciones alternativas y recursos que los participantes tienen a mano. Pero, se diferencia de esta corriente al intentar resaltar las regularidades y patrones en lugar del "anarquismo" (Cohen, March, \& Olsen 1972) con que los teóricos de la lata de basura describen al Estado. Propone superar las dificultades teóricometodológicas en la identificación de los flujos de procesos a partir de la descripción de (1) las formas en que se construye e identifica un problema, (2) la propuesta de alternativas y soluciones al problema; y (3) el humor político que facilita u obstruye su tratamiento final.

Como se adelantó en el apartado anterior, los informáticos en salud tuvieron dificultades para posicionar la falta de disponibilidad de información en tiempo real como un problema de salud durante varias décadas. Los avances en salud digital se dieron de forma desarticulada. A pesar de ser los Estados nacionales los interesados en medir a las poblaciones, el discurso de las estadísticas confiables y la trazabilidad de los datos empleados por la OMS para el desarrollo de SIS no lograron construir marcos interpretativos eficientes para movilizar recursos políticos a nivel nacional. Este asunto logró un cambio cuando el desarrollo de SIS se construyó como una alternativa para la solución de un problema con mayor trayectoria y reconocimiento por parte de la sociedad civil: la desigualdad en el acceso a la salud. Por esta razón se vuelve relevante señalar a la Cobertura Universal como un elemento que finalmente logra el posicionamiento estratégico de la salud digital y la definición de estándares de interoperabilidad.

Ante la necesidad de planificar e implementar programas que colaboren efectivamente en la reducción de la desigualdad en el acceso a la salud, la OPS/ OMS conformó un grupo de trabajo durante 2013 que generó, a partir de un concepto surgido en la Asamblea Mundial de 2005, la estrategia "Cobertura Universal de Salud”. La propuesta contiene cuatro líneas rectoras que buscan (1) ampliar el acceso equitativo a los servicios de salud, (2) fortalecer la rectoría y gobernanza, (3) aumentar el financiamiento y (4) promover la acción intersectorial para abordar los determinantes sociales de la salud (OMS, 2014). El cambio terminológico desde el "acceso" hacia "cobertura universal” despertó polémicas. Para la OMS hablar de cobertura no implica reducir esfuerzos o derechos; ambos términos serían dos caras de una misma moneda. Mientras que el acceso pone el foco en el derecho de los ciudadanos, la cobertura enfatiza la responsabilidad de los Estados en garantizar las prestaciones. Sin embargo, la 
denominación de esta estrategia sumada a la implementación de los programas en algunos países generó críticas y movilización pública.

En el ámbito local, se decidió implementar la CUS a partir de 2016 (Decreto $\left.\mathrm{N}^{\circ} 908,2016\right)$. El Ministerio de Salud nacional intentó evitar las controversias registradas en otros países operando un giro discursivo al señalar que el espíritu de la CUS es la disminución de las desigualdades y no la promoción de una canasta básica de prestaciones. Este posicionamiento se condensó en el slogan "la CUS pretende pasar de una cobertura aspiracional a una real", buscando poner foco en la continuidad de los tratamientos y responder a las críticas que señalaban que Argentina ya cuenta con un sistema público de salud.

Las cuatro líneas estratégicas que propone la CUS de la OPS/OMS se sintetizaron en tres ejes de trabajo a nivel nacional: el primero de ellos hace foco en la atención primaria de la salud desde el marco de la medicina familiar y comunitaria, el segundo se refiere a la salud digital y el tercero a la ampliación de la cobertura y mejora de la atención. El título "salud digital” para el segundo eje fue utilizado en las comunicaciones a públicos amplios, mientras que en los documentos oficiales la referencia más frecuente al eje era "sistemas de información interoperables y aplicaciones informáticas". Esta denominación técnica evidencia ya la elección por la determinación de estándares que "permitan compartir la información entre niveles de atención y jurisdicciones, construyendo para cada paciente una historia clínica nacional, longitudinal y completa, de utilidad clínica, estadística y de gestión" (Resolución $189,2018)$ ante otras alternativas.

La implementación de la CUS en Argentina y la valorización que hizo de los SIS nos llevan a analizar nuevamente el encuadre de los problemas y la búsqueda de soluciones alternativas. La digitalización se presentó como un medio capaz de reducir las desigualdades en el acceso a la salud. A pesar de este marco, la CUS y el desarrollo de SIS recolectaron más críticas que apoyo de sanitaristas y organizaciones sociales (Ortúzar, 2018). La construcción de una red integrada de datos de salud no se configura como prioritaria en un contexto donde los reclamos de la ciudadanía señalan grandes dificultades edilicias, de provisión de insumos y falta de profesionales de salud. Sin embargo, el Estado y los informáticos en salud argumentan que el despliegue de la infraestructura necesaria para soportar la red (conexión a internet, servidores, dispositivos de registro, etc.) asegura atender a estas necesidades y hacerlo de un modo más eficiente. La frase recurrente del Ministerio para sintetizar este concepto fue: "Sistemas de información interoperables como puente a la Cobertura Universal de Salud".

La estrategia CUS ha sido acusada por los sanitaristas y organizaciones sociales de favorecer la mercantilización de la salud (Asociación de Trabajadores del Estado, 2017; Ciciliani, 2016; Lombardi, 2018). En particular, la decisión de optar por la interoperabilidad de los SIS apuntando a mantener la autonomía de las provincias y reconocer los desarrollos públicos y privados preexistentes fue asociado a mantener la "fragmentación" del sistema de salud (Rovere, 2016) mientras se pretende la integración de la información. Así, la interoperabilidad para el Estado supone la solución a diversos problemas como ser el financiamiento de las políticas de salud digital y la reducción de la fricción con las autoridades provinciales y los actores del mercado de la salud. Pero para una parte de la 
opinión pública, significa mantener e incluso incrementar la fragmentación del sistema de salud. De este modo, lejos de entender que se trata de una forma de mejorar las desigualdades en el acceso y calidad de atención, la interoperabilidad exacerbaría la mercantilización de la salud. Para estos sectores, superar la "interoperabilidad de la bolsita" mediante la digitalización del registro y la creación de una red integrada de datos, no asegura que los ciudadanos sean atendidos de modo integral.

Los críticos de la CUS también señalan que la implementación de los SIS está orientada a mejorar la gestión administrativa y optimizar los recursos a discreción política, en lugar de estar orientados a la mejora de la atención. Desde el campo de la informática en salud se responde a estos temores desplegando dos argumentos. El primero de ellos, referencia la construcción de la identidad profesional señalando que son gestores de datos en salud y no administradores.

\footnotetext{
Me di cuenta que la gente empezó a entender qué hace un informático médico (...). Saben que no somos gestores de salud, digamos, que era con lo que nos confundían o nos pueden seguir confundiendo desde algún punto. $\mathrm{O}$ sea, yo no gestiono un hospital, yo gestiono la información del hospital. (...) sí te puedo decir cómo tenés que manejar la información para que a ese paciente le vaya mejor que a otro. Después si en eso se ahorró plata, no sé, no es ese mi foco principal. (Notas de campo, 2017. Entrevista a Informático en Salud).
}

El segundo argumento apunta a señalar que SIS eficientes no solo mejoran la calidad de atención, sino que también permiten hacer un uso eficiente y efectivo de los recursos. La noción de código técnico invita a complejizar el análisis de este argumento y revisar de modo simétrico (Latour, 2008) los efectos de la implementación de una tecnología. Es decir, cada uno de los actores implicados puede poseer un interés particular sobre los beneficios de los SIS y estas implicaciones yuxtaponerse en varios planos. Por ejemplo, las aplicaciones de telecomunicaciones permiten acercar calidad de atención a todo el territorio y permiten a pacientes realizar tratamientos de mayor complejidad sin tener que trasladarse. Ello implica para los financiadores de salud reducir el costo de los traslados de pacientes y/o de profesionales especializados, pero también, la telemedicina puede evitar el desarraigo y mejorar la respuesta a los tratamientos y/o la pronta respuesta ante urgencias (Hess et al., 2005; Nouhi, Fayaz-Bakhsh, Mohamadi \& Shafi, 2012).

A este punto referido a la construcción de alternativas y soluciones a los problemas, correspondiente al segundo flujo de procesos, se asocia la conformación de comunidades de especialistas que se presentan como los expertos para ofrecer soluciones. $\mathrm{Al}$ igual que en el apartado anterior, se vuelve relevante revisar la construcción del perfil profesional de los informáticos. Estos profesionales proponen enmarcar el uso de SIS como parte del proceso asistencial y no como un momento de carga de trabajo administrativa o como una mera transacción de información. Poniendo el foco en el proceso de salud buscan que los debates sobre interoperabilidad no terminen en discusiones técnicas ni en una fetichización de la tecnología. Una de las trabajadoras del Ministerio de Salud Nacional sintetizó la percepción de algunos miembros de los equipos de salud sobre la interoperabilidad y la CUS.

SY comenzó explicando que su punto de vista era desde lo asistencial y que entendía que el desafío era pensar la interoperabilidad en el territorio para cuestiones de 
atención y no de toma de decisiones para la gestión. (...) Ella continuó explicando que lo que había identificado como nuevo en los proyectos de la CUS era pensar la interoperabilidad desde el punto de vista del paciente y al paciente/ ciudadano como la autoridad sobre los datos. (Notas de campo, 2019. Entrevista a trabajadores de la Secretaría de Salud de la Nación).

La innovación que introdujo la CUS para el desarrollo de SIS fue agrupar todos proyectos de desarrollo previos y, como señaló SY, apelar al argumento de que se trata de solucionar un problema de salud. La OPS/OMS busca una mayor participación de los pacientes, de este modo si la visión integral de una historia clínica se puede reconstruir con los ciudadanos en el centro, podrían seguir teniendo el poder sobre la "bolsita". Con control sobre su información, los ciudadanos podrían habilitar a los profesionales el acceso a sus datos, "empoderándose" como dueños de su información clínica y así participar en las decisiones de tratamiento (Lopez Osornio, 2018; Rizzato Lede, 2018).

El tercero de los flujos de procesos que Kingdon propone identificar para interpretar la conformación de la agenda tiene relación con el humor político del momento en que finalmente se abre la ventana de posibilidad que permite legislar sobre tema en cuestión. El principal evento de esta serie de procesos fue el cambio en la administración pública. En 2015, la coalición Cambiemos ganó las elecciones presidenciales y utilizó la digitalización como parte de los discursos sobre la modernización del Estado (Schuttenberg, 2017). Tomando ventaja de las posibilidades que brinda el cambio de una gestión para incorporar nuevos temas en la agenda, la estrategia CUS se presentó como una oportunidad para avanzar con la informatización del sector de la salud. Sumado a los proyectos de digitalización del gobierno, adherir a la CUS facilitaba la asistencia técnica y financiamiento de la OPS/OMS para el sector de la salud.

\section{La apertura de una ventana de posibilidad. Reorganización de la agenda política}

La identificación del tipo de ventana de oportunidad que finalmente permite el tratamiento de un tema como objeto de políticas públicas es una estrategia para articular a los actores y los flujos de procesos. Los dos tipos de ventana que propone Kingdon (2014, pp. 165-195) responden a momentos de crisis o a movimientos políticos. A partir de lo desarrollado anteriormente, argumentamos que el tratamiento de la interoperabilidad de los SIS responde a una ventana del tipo político ya que fue traccionada principalmente por un grupo de interés, los informáticos en salud, en un momento donde se combinó el cambio en el poder ejecutivo y un programa de organismos internacionales con recursos técnicos y financiamiento a disposición. No se trató de la respuesta a una crisis donde los políticos salieron en la búsqueda de soluciones frente a un problema, sino que evaluaron las alternativas disponibles para emplearla en su administración. En el contexto de un cambio de gestión con un discurso que contemplaba, por un lado, la digitalización, y por el otro, la mejora de las relaciones con organismos internacionales, los informáticos en salud movilizaron sus recursos y presentaron propuestas y alternativas. Algunas de las visiones de la comunidad local ya estaban contempladas en la CUS dado que participan como consultores en la OPS/OMS. 
Revisando las relaciones entre los representantes del gobierno involucrados en la implementación de la estrategia CUS y la comunidad de los informáticos en salud, emerge recurrentemente el Hospital Italiano de Buenos Aires. Este centro de salud se posicionó como de vanguardia no solo por el desarrollo e investigación en el campo sino también por la formación de recursos humanos calificados en la disciplina. Este hospital facilitó al Ministerio los recursos para el desarrollo del Bus de Interoperabilidad. También resulta relevante señalar que allí trabajó Adolfo Rubinstein, el segundo médico elegido por la coalición Cambiemos para dirigir la cartera nacional de salud. Su periodo de trabajo asistencial coincide con el proceso de digitalización del hospital. Como "emprendedor político" (Kingdon, 2014, p. 179), logró articular las necesidades del partido que lo convocó, incluir el mandato de la OMS y parte de la agenda de los informáticos en salud.

$\mathrm{Al}$ asumir el poder en diciembre de 2019, la coalición Frente de Todos ha reinstalado la cartera de salud al rango de Ministerio y ha reconfigurado la Dirección Nacional de Sistemas de Información en Salud en la Dirección Nacional de Gobernanza e Integración de los Sistemas de Salud. A pesar del cambio de gobierno, se anunció la continuación de los desarrollos de servicios que sean consumidos por las jurisdicciones y las prestadoras de salud haciendo converger los desarrollos autónomos en modelos comunes. A nivel de las provincias el programa de apoyo a los desarrollos e implementaciones viene dado por la hoja de ruta planteada por la CUS.

La elevación de la interoperabilidad como tema de agenda logró instalarse sin que todavía estén definidos política y técnicamente los modos en que esos datos serían visualizados por los profesionales, o cómo los pacientes autorizarían el acceso a los mismos. Para poder llevar adelante estos proyectos, se debe rever y adecuar aspectos legales y regulatorios de las nuevas prácticas que median los SIS. Esto continúa implicando un desafío para la comunidad que a lo largo de los años no mostró lograr una ágil movilización de sus intereses en la arena política ${ }^{2}$. Esta nueva infraestructura de información en salud a partir de estándares de interoperabilidad que ha sido celebrada y criticada por mantener el statu quo, ha demostrado ser una tecnología relevante en procesos de reconfiguración sociales de escala global (Clarke et al., 2003). Cabría preguntarse si algún otro grupo de interés o crisis logrará enmarcar estos procesos como de cambio social y capturar la atención de la sociedad civil.

\section{Conclusiones}

En este artículo utilizamos el modelo del ciclo de las políticas públicas para analizar el tratamiento de una estrategia de salud digital organizada a partir de la interoperabilidad de los sistemas de información. Argumentamos que el ascenso del tema fue el resultado de la apertura de una ventana de oportunidad del tipo política. La convergencia de un cambio de administración en el poder ejecutivo y la propuesta moderada de la comunidad de los informáticos en salud facilitaron el tratamiento del tópico dentro del marco ofrecido por programas de organismos internacionales.

Los estándares de interoperabilidad son una alternativa de desarrollo técnico y la opción elegida para integrar la información clínica y administrativa de un 
sistema nacional de salud que se mantiene fragmentado. La noción de código técnico nos permitió identificar que la interoperabilidad condensa múltiples sentidos y brinda soluciones de modo virtuoso a necesidades e intereses de varios involucrados. Para la administración nacional la interoperabilidad se presentó como una estrategia para la implementación de la Cobertura Universal de Salud y reducir la desigualdad en el acceso y calidad. Desarrollar un Bus de Interoperabilidad es una de las tecnologías más económicas, ya que el desarrollo y mantenimiento de aplicativos depende de cada prestadora de salud. A la vez, se conserva la descentralización de los servicios asistenciales y se habilita trabajar coordinadamente con las jurisdicciones, el sector de prestaciones privadas y de la seguridad social. Mantener la descentralización significa para un grupo de sanitaristas y organizaciones sociales que la interoperabilidad sostenga y agudice la mercantilización de la salud y el desfinanciamiento del sector público.

Para la comunidad de informáticos en salud, la interoperabilidad permite el desarrollo más eficiente de una red de sistemas de información que puede ser actualizada según las tecnologías disponibles. La interoperabilidad, también, es la tecnología que permitió acercar la comunidad de especialistas de forma efectiva a la política nacional. Asimismo, el consenso sobre los estándares consolidó el trabajo de la comunidad al proveer un marco regulatorio para sus desarrollos. Este último aspecto refleja el reconocimiento de la informática en salud como disciplina y nuevos espacios laborales para el nuevo perfil profesional. Un perfil de especialistas cuya agenda y alternativas excede por mucho a la interoperabilidad y la cobertura universal. Es una agenda que invita a revisar discursos, prácticas y relaciones de poder tradicionales en la medicina.

\section{Agradecimientos}

Este artículo se realizó dentro del marco de una beca doctoral otorgada por la Secretaría de Ciencia y Técnica de la Universidad de Buenos Aires (Res CS 7741, 2017). El trabajo se desarrolla en el Instituto de Ciencias Antropológicas para el proyecto de investigación "Co-producción de conocimiento: nuevos formatos asociativos y materialidad de la creatividad científica” (20020170100593BA), dirigido por la Dra. Cecilia Hidalgo.

\section{Referencias}

Asociación de Trabajadores del Estado. (2017). La CUS es el caballo de Troya para la mercantilización de la Salud.

Berg, M. (1997). Rationalizing medical work. Decision-support techniques and medical practices. MIT Press.

Castaneda, C., Nalley, K., Mannion, C., Bhattacharyya, P., Blake, P., Pecora, A., Goy, A., \& Suh, K. S. (2015). Clinical decision support systems for improving diagnostic accuracy and achieving precision medicine. Journal of Clinical Bioinformatics, 5, 4.

Ciciliani, A. (2016). La mercantilización de la medicina pública a través del CUS. El Cronista.

Clarke, A. E., Shim, J. K., Mamo, L., Fosket, J. R., \& Fishman, J. R. (2003). Biomedicalization: Technoscientific Transformations of Health, Illness, and US Biomedicine. Source: American Sociological Review, 68(2), 161- 194. 
Cohen, M. D., March, J. G., \& Olsen, J. P. (1972). A Garbage Can Model of Organizational Choice. Administrative Science Quarterly, 17(1), 25. https:// doi.org/10.2307/2392088

Edwards, P. N. (2010). A vast machine\#: computer models, climate data, and the politics of global warming. MIT Press.

Epstein, S. (1996). Impure Science: Aids, Activism, and the Politics of Knowledge. University of California Press.

Friedman, C. (2009). A fundamental theorem of biomedical informatics. Journal of the American Medical Informatics Association\#: JAMIA, 16(2), 169- 170.

Gardner, R. M., Overhage, J. M., Steen, E. B., Munger, B. S., Holmes, J. H., Williamson, J. J., Detmer, D. E., \& AMIA Board of Directors, A. B. of. (2009). Core content for the subspecialty of clinical informatics. Journal of the American Medical Informatics Association, 16(2), 153- 157. https://doi.org/10.1197/jamia.M3045

Gunter, T. D., \& Terry, N. P. (2005). The emergence of national electronic health record architectures in the United States and Australia: models, costs, and questions. Journal of Medical Internet Research, 7(1), e3.

Herrero, R., y Guerra, J. (2017). Seguridad de la Información en entornos de salud. HL7 LATAM NEWS.

Hess, D. C., Wang, S., Hamilton, W., Lee, S., Pardue, C., Waller, J. L., Gross, H., Nichols, F., Hall, C., \& Adams, R. J. (2005). REACH: Clinical feasibility of a rural telestroke network. Stroke, 36(9), 2018- 2020. https:// doi.org/10.1161/01.STR.0000177534.02969.e4

Jasanoff, S. (2004). States of Knowledge: The Co-Production of Science and the Social Order (S. Jasanoff (ed.)). Routledge.

Jasanoff, S., \& Kim, S. H. (2013). Sociotechnical Imaginaries and National Energy Policies. Policy Imaginations, 22(2), 189-196. https:// doi.org/10.1080/09505431.2013.786990

Jasanoff, S., \& Simmet, H. R. (2017). No funeral bells: Public reason in a 'post-truth' age. Social Studies of Science, 47(5), 751-770. https:// doi.org/10.1177/0306312717731936

Kingdon, J. W. (2014). Agendas, Alternatives, and Public Policies (2o). Oxford University Press.

Latour, B. (2008). Reensamblar lo social. Manantial.

Lindberg, D. A. B. (1965). Electronic Retrieval of Clinical Data Electronic Retrieval of Clinical Data. The Journal of Medical Education, 40(8).

Lombardi, V. (2018). Seguro de salud: ¿Una puerta a la privatización del sistema sanitario? Agencia TSS.

Lopez Castro, M. B., Smulski, M. C., \& Hidalgo, C. (2019). Inquietud y contingencia. Tres casos de "enredos experimentales" en la investigación ID-TD. Climacom, $5(13)$.

Lopez Osornio, A. (2018). Estrategia Nacional de Salud Digital.Jornadas Universitarias de Sistemas de Información en Salud.

Luna, D., Otero, C., Almerares, A., Stanziola, E., Risk, M., \& González Bernaldo de Quirós, F. (2015). Participatory design for drug-drug interaction alerts. Studies in Health Technology and Informatics, 210, 45- 49.

Mantas, J., Ammenwerth, E., Demiris, G., Hasman, A., Haux, R., Hersh, W., Hovenga, E., Lun, K. C., Marin, H., Martin-Sanchez, F., Wright, G., \& IMIA Recommendations on Education Task Force. (2010). Recommendations of the International Medical Informatics Association on Education in Biomedical 
and Health Informatics. Methods of Information in Medicine, 49(2), 105-120. https://doi.org/10.3414/ME5119

Nouhi, M., Fayaz-Bakhsh, A., Mohamadi, E., \& Shafii, M. (2012). Telemedicine and its potential impacts on reducing inequalities in access to health manpower. Telemedicine and E-Health, 18(8), 648- 653. https://doi.org/10.1089/ TMJ.2011.0242

Ortúzar, M. G. de. (2018). Cobertura Universal de Salud -CUS- Vs Derecho a la Salud. Revista de Ciencias Sociales y Humanas, 12(12), 103- 116.

Oszlak, O. (2006). Burocracia estatal: política y políticas públicas. POSTData. Revista de Reflexión y Análisis Político, 11- 56.

Oszlak, O., y Gantman, E. (2007). La agenda estatal y sus tensiones: gobernabilidad, desarrollo y equidad. Iberoamericana. Nordic Journal of Latin American and Caribbean Studies, 37(1), 79- 110.

Oszlak, O., \& O’Donnell, G. (1995). Estado y políticas estatales en América Latina: hacia una estrategia de investigación. REDES, 2(4), 99- 128.

Rizzato Lede, D. (2018). Estrategia Nacional de Salud Digital. Congreso Argentino de Informática y Salud.

Rovere, M. (2016). El Sistema de Salud de la Argentina como Campo; Tensiones, Estratagemas y Opacidades. Debate Público Reflexión de Trabajo Social, 6(12), 2341.

Schuttenberg, M. (2017). La política de la despolitización. Un análisis de la construcción del relato PRO. Desafios, 29(2).

Simborg, D. W. (1988). Network Application Architecture. Proceedings of the Annual Symposium on Computer Application in Medical Care, 648.

Wegner, P. (1996). Interoperability. ACM Computing Surveys, 28(1), 285- 287. https://doi.org/10.1145/234313.234424

Winner, L. (1980). Do Artifacts Have Politics? Daedalus, 109(1), 121- 136.

\section{Fuentes}

Resolución 883 de 2007. [Ministerio de Salud]. Boletín Oficial de la República Argentina, 7 de agosto de 2007.

Decreto 908 de 2016. [Ministerio de Salud]. Cobertura Universal de Salud. Fodo Solidario de Redistribución. Boletín Oficial de la República Argentina, 2 de agosto de 2016.

Decisión Administrativa 307 de 2018. [Ministerio de Salud]. Boletín Oficial de la República Argentina, 13 de marzo 2018.

Resolución 189 de 2018. Ministerio de Salud y Desarrollo Social]. Estrategia Nacional de Salud Digital 2018-2024. Boletín Oficial de la República Argentina, 30 de octubre de 2018.

Resolución 115 de 2019. [Ministerio de Salud y Desarrollo Social]. Boletín Oficial de la República Argentina 24 de enero de 2019.

Resolución 680 de 2020. [Ministerio de Salud]. Boletín Oficial de la República Argentina 30 de marzo de 2020. 


\section{Notas}

1. Por ejemplo, IRA podría referirse a un estado emocional, o ser una abreviatura para una infección respiratoria o de una insuficiencia renal aguda.

2. Las dificultades de esta movilización, especialmente sobre el parlamento se evidenciaron durante la pandemia de la covid-19. Ante la crisis se logró consenso para legislar y regularizar los marcos normativos de prácticas como la telemedicina y las recetas electrónicas, dos proyectos que venían siendo postergados desde hacía tres años. 